\title{
The distribution of squarefull numbers in short intervals
}

\author{
by \\ Michael Filaseta (Columbia, S.C.) and Ognian Trifonov (Sofia)
}

1. Introduction. A squarefull number is a positive integer $n$ such that if $p$ is a prime dividing $n$, then $p^{2}$ divides $n$. Let $Q(x)$ denote the number of $n \leq x$ which are squarefull. Then a result of Bateman and Grosswald [1] implies that

$$
Q(x)=\frac{\zeta(3 / 2)}{\zeta(3)} x^{1 / 2}+\frac{\zeta(2 / 3)}{\zeta(2)} x^{1 / 3}+o\left(x^{1 / 6}\right) .
$$

It follows that there is a positive constant $c_{1}$ and arbitrarily large values of $x$ for which the interval $\left(x, x+c_{1} x^{1 / 2}\right]$ contains no squarefull numbers. On the other hand, since there is a square in $(x, x+2 \sqrt{x}+1]$ for all $x \geq$ 0 , it follows that there is a constant $c_{2}$ such that for every $x \geq 1$, the interval $\left(x, x+c_{2} x^{1 / 2}\right]$ contains a squarefull number. Thus, the order of the maximum size of gaps between squarefull numbers is known. However, a better understanding of the distribution of squarefull numbers in short intervals can still be obtained. The result of Bateman and Grosswald easily implies that

$$
Q\left(x+x^{1 / 2+\theta}\right)-Q(x) \sim \frac{\zeta(3 / 2)}{2 \zeta(3)} x^{\theta}
$$

provided $1 / 6 \leq \theta<1 / 2$. It is not too difficult to see that if (1) holds for some $\theta=\phi \in(0,1 / 2)$, then it holds for every fixed $\theta \in[\phi, 1 / 2)$. Hence, we would like to determine the smallest value of $\theta \in(0,1 / 2)$ for which (1) holds. It is likely that any $\theta>0$ will do. Values of $\theta<1 / 6$ which have been obtained include $0.1526 \ldots$ by Shiu [10], $0.1507 \ldots$ by P. G. Schmidt [8], $0.14903 \ldots$ by Jia [6], $0.142857 \ldots$ by P. G. Schmidt [9], $0.14254 \ldots$ by Liu [7], and $0.1317 \ldots$ by Heath-Brown [4]. In particular, Heath-Brown made a

Research of the first author supported by the NSA under grant number MDA904-92H-3011.

Research of the second author supported by NSF EPSCoR Grant EHR 9108772 and by the Bulgarian National Research Foundation, contract MM27. 
remarkable improvement on previous results by considering

$$
S(x)=\sum_{a^{2} b^{3} \leq x} 1
$$

and showing that asymptotic estimates for $S\left(x+x^{1 / 2} y\right)-S(x)$ imply similar asymptotic estimates for $Q\left(x+x^{1 / 2} y\right)-Q(x)$ (see Lemma 1 below). Using a result of P. G. Schmidt [8], it follows from Heath-Brown's work that one can take in (1) any $\theta$ satisfying $27 / 205=0.1317 \ldots<\theta<1 / 2$. We show how to take advantage of finite difference techniques to obtain

TheOREM. The asymptotic formula in (1) holds for $5 / 39=0.1282 \ldots<$ $\theta<1 / 2$.

2. Preliminaries. We begin here with a slight reformulation of HeathBrown's result in [4]. Although, we could proceed by using his results directly, we alter them slightly so as to give an elementary argument of the main result in this paper so that, in particular, our results do not depend on the use of exponential sums. We shall refer to the result of Heath-Brown in [4] and to a result of Huxley in [5], and note here that the arguments for both of these are obtained using elementary techniques.

Lemma 1. Let $\theta \in(0,1 / 2)$ and let $\varepsilon \in(0, \theta)$. Suppose there is a $\delta=$ $\delta(\theta, \varepsilon)>0$ such that

$$
S\left(x+x^{1 / 2+\gamma}\right)-S(x)=\frac{\zeta(3 / 2)}{2} x^{\gamma}\left(1+O\left(x^{-\delta}\right)\right)
$$

uniformly for $\theta-\varepsilon \leq \gamma \leq \theta$. Then there is an $\eta=\eta(\theta, \varepsilon)>0$ such that

$$
Q\left(x+x^{1 / 2+\theta}\right)-Q(x)=\frac{\zeta(3 / 2)}{2 \zeta(3)} x^{\theta}\left(1+O\left(x^{-\eta}\right)\right) .
$$

To clarify the statement of Lemma 1 and the role of $\gamma$ throughout the paper, we note that the uniformity condition on $\gamma$ indicates that the implied constant in the displayed formula involving $S(x)$ is absolute provided $x \geq$ $x_{0}=x_{0}(\theta, \varepsilon)$. This formula is to hold for all $\gamma$ with

$$
x^{\theta-\varepsilon} \leq x^{\gamma} \leq x^{\theta}
$$

so that, for example, we will allow $\gamma=\theta-\log \log x / \log x$. The proof of Lemma 1 is essentially the same as the proof of the Theorem of Heath-Brown in [4]. Simply take

$$
\theta_{0}=\frac{(\theta-\varepsilon)(1-2 \theta)}{1-2 \theta+2 \varepsilon}
$$

in his argument (not in his Theorem). To estimate $S\left(x+x^{1 / 2+\gamma}\right)-S(x)$, we make use of the following. 
Lemma 2. Let $x$ and $z$ denote positive real numbers. Let $c \in(0,1 / 4)$, and let $h=\sqrt{x} y$ where $y=x^{\gamma}$ and $c<\gamma<1 / 2-c$. Fix $\delta>0$ with $\delta$ sufficiently small (possibly depending on $c$ but not depending on $\gamma$ ). Then

where

$$
S(x+h)-S(x)=\frac{\zeta(3 / 2)}{2} y+O\left(S_{1}\right)+O\left(S_{2}\right)+O\left(y x^{-\delta}\right),
$$

and

$$
S_{1}=S_{1}(z)=\sum_{y x^{-\delta}<n \leq z}\left(\left[\sqrt[3]{\frac{x+h}{n^{2}}}\right]-\left[\sqrt[3]{\frac{x}{n^{2}}}\right]\right)
$$

$$
S_{2}=S_{2}(z)=\sum_{y x^{-\delta}<n \leq 2 x^{1 / 3} z^{-2 / 3}}\left(\left[\sqrt{\frac{x+h}{n^{3}}}\right]-\left[\sqrt{\frac{x}{n^{3}}}\right]\right) .
$$

Proof. Let $z_{0}$ be defined by $y x^{-\delta}=2 x^{1 / 3} z_{0}^{-2 / 3}$ so that $y x^{-\delta} \leq 2 x^{1 / 3} z^{-2 / 3}$ if and only if $z \leq z_{0}$. If $z>z_{0}$, then $S_{1}(z) \geq S_{1}\left(z_{0}\right)$ and $S_{2}(z)=S_{2}\left(z_{0}\right)=0$. Thus, if the lemma holds for $z \leq z_{0}$, then the lemma holds for $z>z_{0}$ as well. Similarly, one checks that if the lemma holds for $z \geq y x^{-\delta}$, then it holds for $z<y x^{-\delta}$ as well. Therefore, we only consider

Observe that

$$
y x^{-\delta} \leq z \leq z_{0} .
$$

$$
S(x+h)-S(x)=\sum_{x<a^{2} b^{3} \leq x+h} 1 .
$$

On the other hand, if $x<a^{2} b^{3} \leq x+h$, then it is easy to see that either $a \leq z$ or $b \leq 2 x^{1 / 3} z^{-2 / 3}$. First, we consider all such pairs $(a, b)$ with $b \leq$ $2 x^{1 / 3} z^{-2 / 3}$. Note that for each $b \leq 2 x^{1 / 3} z^{-2 / 3}$, we have

$$
\sqrt{\frac{x}{b^{3}}}<a \leq \sqrt{\frac{x+h}{b^{3}}} ;
$$

hence, the total number of such pairs is

$$
\begin{aligned}
\sum_{n \leq 2 x^{1 / 3} z^{-2 / 3}}\left(\left[\sqrt{\frac{x+h}{n^{3}}}\right]-\left[\sqrt{\frac{x}{n^{3}}}\right]\right) \\
=\sum_{n \leq y x^{-\delta}}\left(\left[\sqrt{\frac{x+h}{n^{3}}}\right]-\left[\sqrt{\frac{x}{n^{3}}}\right]\right)+S_{2} \\
=\sum_{n \leq y x^{-\delta}}\left(\sqrt{\frac{x+h}{n^{3}}}-\sqrt{\frac{x}{n^{3}}}\right)+S_{2}+O\left(y x^{-\delta}\right) \\
=\frac{\zeta(3 / 2)}{2} y-\frac{y}{2} \sum_{n>y x^{-\delta}} \frac{1}{n^{3 / 2}}+S_{2}+O\left(y x^{-\delta}\right) \\
=\frac{\zeta(3 / 2)}{2} y+S_{2}+O\left(y x^{-\delta}\right) .
\end{aligned}
$$


Now, we count the pairs $(a, b)$ with $a \leq z$. We may be counting some pairs here that we have already counted, but observe that in any case we are through if we can show that the number of such pairs is $O\left(S_{1}\right)+O\left(y x^{-\delta}\right)$. For each such pair, we have

$$
\sqrt[3]{\frac{x}{a^{2}}}<b \leq \sqrt[3]{\frac{x+h}{a^{2}}}
$$

Thus, the number of such pairs is bounded above by

$$
\begin{aligned}
\sum_{n \leq z}\left(\left[\sqrt[3]{\frac{x+h}{n^{2}}}\right]\right. & \left.-\left[\sqrt[3]{\frac{x}{n^{2}}}\right]\right) \\
& =\sum_{n \leq y x^{-\delta}}\left(\left[\sqrt[3]{\frac{x+h}{n^{2}}}\right]-\left[\sqrt[3]{\frac{x}{n^{2}}}\right]\right)+S_{1} \\
& =\sum_{n \leq y x^{-\delta}}\left(\sqrt[3]{\frac{x+h}{n^{2}}}-\sqrt[3]{\frac{x}{n^{2}}}\right)+S_{1}+O\left(y x^{-\delta}\right) \\
& =S_{1}+O\left(y x^{-\delta}\right),
\end{aligned}
$$

and the result follows.

It remains to estimate $S_{1}$ and $S_{2}$ for an appropriate choice of $z$. To obtain our theorem from Lemma 1 , we take $\theta=5 / 39+\varepsilon^{\prime}$ with $\varepsilon^{\prime}>0$ and we take $\varepsilon$ in Lemma 1 so that $0<\varepsilon<\varepsilon^{\prime}$. As mentioned in the introduction, to obtain the full range of $\theta$ stated in the theorem, it suffices to consider $\varepsilon^{\prime}$ sufficiently small. In particular, in Lemma 1, we need only consider

$$
5 / 39+\left(\varepsilon^{\prime}-\varepsilon\right) \leq \gamma \leq 5 / 39+\varepsilon^{\prime} \leq 9 / 67
$$

This restriction on $\gamma$ is only made to keep the arguments simpler and more self-contained; since $27 / 205<9 / 67$, we could have appealed to the work of P. G. Schmidt [9] to deal with $\gamma>9 / 67$.

In Lemma 2, we take

$$
c=5 / 39, \quad \delta=\frac{1}{2}\left(\varepsilon^{\prime}-\varepsilon\right) \quad \text { and } \quad z=x^{3 / 13} .
$$

The theorem will follow if we can show that $S_{1}\left(x^{3 / 13}\right)$ and $S_{2}\left(x^{3 / 13}\right)$ are each $\ll x^{5 / 39} \log x$. We define

$$
S_{1}(\alpha, \beta)=\sum_{\alpha<n \leq \beta}\left(\left[\sqrt[3]{\frac{x+h}{n^{2}}}\right]-\left[\sqrt[3]{\frac{x}{n^{2}}}\right]\right) .
$$

To estimate $S_{1}$, we consider first $S_{1}(N, 2 N)$ where $y x^{-\delta} \leq N \leq z$. One can interpret $S_{1}(N, 2 N)$ as the number of pairs $(a, b)$ for which

$$
N<a \leq 2 N \quad \text { and } \quad x<a^{2} b^{3} \leq x+h .
$$


Since $h \leq x$, the last inequality easily implies $b \leq 2 \sqrt[3]{x}$. Hence,

$$
a^{2}(b+1)^{3}-a^{2} b^{3} \geq 3 a^{2} b^{2}=\frac{3 a^{2} b^{3}}{b}>\frac{3 x}{b}>x^{2 / 3}>x^{1 / 2+\gamma}=h .
$$

Therefore, for each $a \in(N, 2 N]$, there is at most one pair $(a, b)$ as above. In other words, $S_{1}(N, 2 N)$ is the number of $a \in(N, 2 N]$ with the property that there is an integer $b$ between $\sqrt[3]{x / a^{2}}$ and $\sqrt[3]{(x+h) / a^{2}}$. For $a \in(N, 2 N]$, we have

$$
\sqrt[3]{\frac{x+h}{a^{2}}}-\sqrt[3]{\frac{x}{a^{2}}} \leq \frac{h}{3 x^{2 / 3} a^{2 / 3}}<(1 / 3) x^{\gamma-1 / 6} N^{-2 / 3}
$$

We set

$$
\delta^{\prime}=(1 / 3) x^{\gamma-1 / 6} N^{-2 / 3} .
$$

Thus, $S_{1}(N, 2 N)$ is bounded by the number of $a \in(N, 2 N]$ such that $\sqrt[3]{x / a^{2}}$ is within $\delta^{\prime}$ of an integer. We use Theorem 3 in Huxley's paper [5]. In the notation there, we set $F(x)=(x+1)^{-2 / 3}, L=M=N, \delta=\delta^{\prime}, T=$ $x^{1 / 3} M^{-2 / 3}$, and $\Delta=x^{1 / 3} N^{-8 / 3}$. One can easily check that all the conditions of the theorem are satisfied. We deduce from this result that

$$
S_{1}(N, 2 N) \ll x^{5 / 36} N^{-1 / 11}+x^{1 / 15} N^{4 / 15} \log x
$$

for $x^{5 / 39} \leq N \leq x^{3 / 13}$. Thus,

$$
\begin{aligned}
S_{1}\left(x^{3 / 13}\right) & =\sum_{y x^{-\delta}<n \leq x^{3 / 13}}\left(\left[\sqrt[3]{\frac{x+h}{n^{2}}}\right]-\left[\sqrt[3]{\frac{x}{n^{2}}}\right]\right) \\
& \ll x^{5 / 36} x^{(-1 / 11)(5 / 39)}+x^{1 / 15} x^{(4 / 15)(3 / 13)} \log x \ll x^{5 / 39} \log x .
\end{aligned}
$$

Our choice of $z=x^{3 / 13}$ focuses our attention on the problem at hand; we must now estimate $S_{2}\left(x^{3 / 13}\right)$. This particular choice of $z$, however, is not so important. We could, for example, have chosen $z=1$ so that $S_{1}(z)=0$. Then we could use Theorem 2 of [5] to obtain an estimate for

$$
\sum_{2 x^{7 / 39}<n \leq 2 x^{1 / 3}}\left(\left[\sqrt{\frac{x+h}{n^{3}}}\right]-\left[\sqrt{\frac{x}{n^{3}}}\right]\right),
$$

thereby establishing part of an estimate for $S_{2}(1)$. The rest of the argument would be the same as what appears in the next section.

Before leaving this section, we note that it is possible to improve on the result of Heath-Brown for gaps between squarefull numbers by combining the above estimate for $S_{1}\left(x^{3 / 13}\right)$ with an estimate for $S_{2}\left(x^{3 / 13}\right)$ obtainable through the use of exponential sums. Using the current theory of exponential sums to estimate $S_{2}\left(x^{3 / 13}\right)$, however, seems to lead to a weaker result than our theorem. 
3. The use of differences. We now use differencing techniques to estimate $S_{2}$. In fact, differencing techniques were already used in our estimate of $S_{1}$, as the result of Huxley that we used was based on differences. Our approach here is motivated by the authors' own work in [2] and [3].

Observe that

$$
2 x^{1 / 3} z^{-2 / 3}=2 x^{7 / 39} .
$$

One can interpret the sum $S_{2}$ appearing in Lemma 2 as the number of positive integral pairs $(a, b)$ for which $a^{2} b^{3} \in(x, x+h]$ and

$$
x^{5 / 39} \leq y x^{-\delta}<b \leq 2 x^{7 / 39} .
$$

For such $(a, b)$, we have

$$
a \leq \frac{\sqrt{x+h}}{b^{3 / 2}}<2 \sqrt{x} x^{-5 / 26}=2 x^{4 / 13} .
$$

Since

$$
(a+1)^{2} b^{3}-a^{2} b^{3}>2 a b^{3}=\frac{2 a^{2} b^{3}}{a}>\frac{2 x}{a}>x^{9 / 13}>x^{1 / 2+\gamma},
$$

we deduce that each $b$ in the range above corresponds to at most one pair $(a, b)$ counted by $S_{2}$. Let $S$ be the set of numbers $b$ for which $a^{2} b^{3}$ is in the interval $(x, x+h]$ for some integer $a$, and let

$$
S_{2}(\alpha, \beta)=\{u \in S: \alpha<u \leq \beta\} .
$$

Then for $x^{5 / 39} \leq N \leq x^{7 / 39}$,

$$
\left|S_{2}(N, 2 N)\right|=\sum_{N<n \leq 2 N}\left(\left[\sqrt{\frac{x+h}{n^{3}}}\right]-\left[\sqrt{\frac{x}{n^{3}}}\right]\right) .
$$

Thus, to estimate the size of $S_{2}$, we can make use of estimates for the size of $\left|S_{2}(N, 2 N)\right|$. We consider $N=x^{\phi}$. We refer to $S_{2}(N, 2 N)$ in this case as $S_{\phi}$. Our theorem then is an easy consequence of the next lemma.

Lemma 3. For $\gamma \leq 9 / 67$ and $\phi \leq 2 / 11$,

$$
\left|S_{\phi}\right| \ll x^{(3 \phi+1) / 12}+y^{1 / 4} x^{(1-2 \phi) / 8} .
$$

Equivalently,

$$
\left|S_{\phi}\right| \ll \begin{cases}x^{(3 \phi+1) / 12} & \text { for } \phi \geq(6 \gamma+1) / 12 \\ y^{1 / 4} x^{(1-2 \phi) / 8} & \text { for } \phi<(6 \gamma+1) / 12 .\end{cases}
$$

Proof. Let $T(a, b)$ denote the set of $u$ for which $u, u+a$, and $u+a+b$ are consecutive elements of $S_{\phi}$, and set $t(a, b)=|T(a, b)|$. Let $D=\operatorname{gcd}(a, b)$. Let

$$
A=\min \left\{x^{(9 \phi-1) / 12}, y^{-1 / 4} x^{(10 \phi-1) / 8}\right\},
$$

and observe that there are $\ll x^{\phi} / A$ occurrences of consecutive $u, u+a$, and $u+a+b$ in $S_{\phi}$ with $\max \{a, b\} \geq A$. Now, suppose $\max \{a, b\}<A$. Note that 
$7 \phi / 24 \geq(9 \phi-1) / 12$ so that $a$ and $b$ are $<x^{7 \phi / 24}$. In particular, for $u \in S_{\phi}$, $a$ and $b$ may be viewed as being much smaller than $u$. We find a bound for $t(a, b)$. We use $m_{j}$ to denote integers. For each $u \in S_{\phi}$, there is an integer $m_{1}$ for which $x<u^{3} m_{1}^{2} \leq x+h$ so that

$$
\frac{\sqrt{x}}{u^{3 / 2}}<m_{1} \leq \frac{\sqrt{x+h}}{u^{3 / 2}}=\frac{\sqrt{x}}{u^{3 / 2}}+O\left(y x^{-3 \phi / 2}\right) .
$$

In other words,

$$
\frac{\sqrt{x}}{u^{3 / 2}}=m_{1}+O\left(y x^{-3 \phi / 2}\right)
$$

Let $f(u)=\sqrt{x} / u^{3 / 2}$, and consider $u \in T(a, b)$. Thus, each of $f(u), f(u+a)$, and $f(u+a+b)$ is within $O\left(y x^{-3 \phi / 2}\right)$ of an integer. Now, there are $\xi_{1} \in$ $(u, u+a)$ and $\xi_{2} \in(u+a, u+a+b)$ for which

$$
f(u+a)-f(u)=a f^{\prime}(u)+\frac{a^{2}}{2} f^{\prime \prime}(u)+\frac{a^{3}}{6} f^{\prime \prime \prime}(u)+O\left(a^{4} f^{(4)}\left(\xi_{1}\right)\right)
$$

and

$$
\begin{aligned}
& f(u+a+b)-f(u+a) \\
& \quad=b f^{\prime}(u+a)+\frac{b^{2}}{2} f^{\prime \prime}(u+a)+\frac{b^{3}}{6} f^{\prime \prime \prime}(u+a)+O\left(b^{4} f^{(4)}\left(\xi_{2}\right)\right) .
\end{aligned}
$$

Since $u \in\left(x^{\phi}, 2 x^{\phi}\right]$ and $a$ and $b$ are less than $u$, we get for each $j \in\{1,2\}$,

$$
f^{(4)}\left(\xi_{j}\right) \asymp x^{(1-11 \phi) / 2} .
$$

Using similar expressions to (2) and (3) for the differences $f^{\prime}(u+a)-f^{\prime}(u)$, $f^{\prime \prime}(u+a)-f^{\prime \prime}(u)$, and $f^{\prime \prime \prime}(u+a)-f^{\prime \prime \prime}(u)$ and considering the sum of the product of (2) with the integer $-b / D$ with the product of (3) with the integer $a / D$, we obtain

$$
\begin{aligned}
m_{2}= & \frac{15 a b(a+b) \sqrt{x}}{8 D u^{7 / 2}}-\frac{35 a b(a+b)(2 a+b) \sqrt{x}}{16 D u^{9 / 2}} \\
& +O\left(a b(a+b)^{3} x^{(1-11 \phi) / 2} D^{-1}\right)+O\left((a+b) D^{-1} y x^{-3 \phi / 2}\right),
\end{aligned}
$$

for some integer $m_{2}$. Since $m_{2}$ is an integer, the first term on the right is at least $1 / 2$ if each other term on the right has absolute value less than $1 / 4$ of the first term. Hence,

$$
a b(a+b) \gg x^{(7 \phi-1) / 2} D
$$

provided

$$
2 \gamma+4 \phi<1
$$

an inequality which holds for the range of $\gamma$ and $\phi$ given in the lemma. Note that the above implies that at least one of any two consecutive differences between elements of $S_{\phi}$ must be $\gg x^{(7 \phi-1) / 6}$. By considering every other 
element of $S_{\phi}$ rather than every element of $S_{\phi}$, we may use the estimate

$$
\min \{a, b\} \gg x^{(7 \phi-1) / 6}
$$

in other words, if necessary, we may estimate instead of $\left|S_{\phi}\right|$ the size of the set

$$
S_{\phi}^{\prime}=\left\{u \in S_{\phi}: \text { if } u+a \in S_{\phi}, \text { then } a \geq \varepsilon x^{(7 \phi-1) / 6}\right\}
$$

for some appropriate $\varepsilon>0$ so that $\left|S_{\phi}\right| \leq 2\left|S_{\phi}^{\prime}\right|+1$ (and we estimate $\left|S_{\phi}^{\prime}\right|$ by redefining $T(a, b)$ in terms of $S_{\phi}^{\prime}$ rather than $\left.S_{\phi}\right)$. Now, suppose that there is a $c$ such that $u$ and $u+c$ are in $T(a, b)$ and $c<\varepsilon x^{\phi}$, where $\varepsilon>0$ is sufficiently small (depending on the arguments which follow). Since $u, u+a$, and $u+a+b$ are consecutive elements in $S_{\phi}$ and $u+c$ is in $S_{\phi}$, we get $c \geq a+b$. Considering $f(u+c+a)-f(u+c)-f(u+a)+f(u)$, we see that there is an integer $m_{3}$ such that

$$
\begin{aligned}
m_{3}= & \frac{15 a c \sqrt{x}}{4 u^{7 / 2}}-\frac{105 a c(a+c) \sqrt{x}}{16 u^{9 / 2}}+\frac{315 a c\left(2 a^{2}+3 a c+2 c^{2}\right) \sqrt{x}}{64 u^{11 / 2}} \\
& +O\left(a c^{4} x^{(1-13 \phi) / 2}\right)+O\left(y x^{-3 \phi / 2}\right) .
\end{aligned}
$$

Similarly, considering $f(u+c+a+b)-f(u+c)-f(u+a+b)+f(u)$, there is an integer $m_{4}$ such that

$$
\begin{aligned}
m_{4}= & \frac{15(a+b) c \sqrt{x}}{4 u^{7 / 2}}-\frac{105(a+b) c(a+b+c) \sqrt{x}}{16 u^{9 / 2}} \\
& +\frac{315(a+b) c\left(2(a+b)^{2}+3(a+b) c+2 c^{2}\right) \sqrt{x}}{64 u^{11 / 2}} \\
& +O\left((a+b) c^{4} x^{(1-13 \phi) / 2}\right)+O\left(y x^{-3 \phi / 2}\right) .
\end{aligned}
$$

We use the fact that there are integers $k_{1}$ and $k_{2}$ such that

$$
a k_{1}+(a+b) k_{2}=D \quad \text { and } \quad \max \left\{\left|k_{1}\right|,\left|k_{2}\right|\right\} \leq(a+b) / D \text {. }
$$

Observe that

$$
k_{1} a c(a+c)+k_{2}(a+b) c(a+b+c)=D c^{2}+O\left(c(a+b)^{3} / D\right)
$$

and

$$
\begin{aligned}
k_{1} a c\left(2 a^{2}+3 a c+2 c^{2}\right)+k_{2}(a+b) c\left(2(a+b)^{2}+3(a+b) c+2 c^{2}\right) \\
=2 D c^{3}+O\left(c^{2}(a+b)^{3} / D\right) .
\end{aligned}
$$

We deduce that

$$
\begin{aligned}
k_{1} m_{3} & +k_{2} m_{4} \\
= & \frac{15 D c \sqrt{x}}{4 u^{7 / 2}}+O\left(D c^{2} x^{(1-9 \phi) / 2}\right)+O\left(c(a+b)^{3} D^{-1} x^{(1-9 \phi) / 2}\right) \\
& +O\left(D c^{3} x^{(1-11 \phi) / 2}\right)+O\left(c^{2}(a+b)^{3} D^{-1} x^{(1-11 \phi) / 2}\right) \\
& +O\left(c^{4}(a+b)^{2} D^{-1} x^{(1-13 \phi) / 2}\right)+O\left((a+b) D^{-1} y x^{-3 \phi / 2}\right) .
\end{aligned}
$$


Using the inequalities $c \geq a+b, a+b \leq 2 x^{7 \phi / 24}$ and $c \leq \varepsilon x^{\phi}$, it is easy to check that every term on the right-hand side of (5) after the first has absolute value $<1 / 7$ of the first term on the right with the possible exception of $O\left(c^{4}(a+b)^{2} D^{-1} x^{(1-13 \phi) / 2}\right)$. It follows that the remaining error term in absolute value is less than $1 / 7$ of the first term or

$$
(a+b)^{2 / 3} c \gg x^{\phi} \text {. }
$$

In the first case, since $k_{1} m_{3}+k_{2} m_{4}$ is an integer, we deduce that

$$
c D \gg x^{(7 \phi-1) / 2} \text {. }
$$

Observe that if (6) holds, then since $a+b \leq 2 A \ll x^{(9 \phi-1) / 12}$ and $\phi \leq 2 / 11$,

$$
c \gg x^{\phi}(a+b)^{-2 / 3} \gg x^{(9 \phi+1) / 18} \gg x^{(7 \phi-1) / 2} .
$$

Thus, in any case, (7) holds. Now, we see from our previous inequality for $a b(a+b)$ that

$$
a b(a+b) c \gg x^{7 \phi-1} .
$$

Recall (4) holds for some integer $m_{2}$. In a similar manner, we deduce that there is an integer $m_{5}$ such that

$$
\begin{aligned}
m_{5}= & \frac{15 a b(a+b) \sqrt{x}}{8 D(u+c)^{7 / 2}}-\frac{35 a b(a+b)(2 a+b) \sqrt{x}}{16 D(u+c)^{9 / 2}} \\
& +O\left(a b(a+b)^{3} x^{(1-11 \phi) / 2} D^{-1}\right)+O\left((a+b) D^{-1} y x^{-3 \phi / 2}\right) .
\end{aligned}
$$

Therefore,

$$
\begin{aligned}
m_{2}-m_{5}= & \frac{105 a b(a+b) c \sqrt{x}}{16 D u^{9 / 2}}+O\left(a b(a+b) c^{2} x^{(1-11 \phi) / 2} D^{-1}\right) \\
& +O\left((a+b) D^{-1} y x^{-3 \phi / 2}\right) .
\end{aligned}
$$

Using the inequality $c \leq \varepsilon x^{\phi}$ and our lower bound for $a b(a+b) c$ above, we conclude that the absolute value of each of the error terms on the right is less than $1 / 3$ of the first term on the right unless

$$
c \ll y x^{(6 \phi-1) / 2} / a b .
$$

Thus, either (8) holds or

$$
\frac{105 a b(a+b) c \sqrt{x}}{16 D u^{9 / 2}} \gg 1
$$

so that

$$
c \gg \frac{D x^{(9 \phi-1) / 2}}{a b(a+b)} .
$$

Recalling that $a b(a+b) \gg x^{(7 \phi-1) / 2} D$, we see that the elements of $T(a, b)$ can be separated into 
$\ll \frac{x^{\phi} a b(a+b)}{D x^{(9 \phi-1) / 2}}+1 \ll a b(a+b) D^{-1} x^{(1-7 \phi) / 2}+1 \ll a b(a+b) D^{-1} x^{(1-7 \phi) / 2}$

subintervals, with distinct elements in each subinterval being within $O\left(y x^{(6 \phi-1) / 2} / a b\right)$ of one another. On the other hand, we have already established that (7) holds so that distinct elements in each subinterval are also separated by $\gg x^{(7 \phi-1) / 2} / D$. In other words, each such subinterval contains

$$
\ll \frac{y x^{(6 \phi-1) / 2} D}{a b x^{(7 \phi-1) / 2}}+1 \ll a^{-1} b^{-1} D y x^{-\phi / 2}+1
$$

elements. We deduce that

$$
\begin{aligned}
t(a, b) & \ll a b(a+b) D^{-1} x^{(1-7 \phi) / 2}\left(a^{-1} b^{-1} D y x^{-\phi / 2}+1\right) \\
& \ll(a+b) y x^{(1-8 \phi) / 2}+a b(a+b) D^{-1} x^{(1-7 \phi) / 2} \\
& \ll(a+b) y x^{(1-8 \phi) / 2}+a b(a+b) x^{(1-7 \phi) / 2} .
\end{aligned}
$$

Observe that $A \leq x^{(9 \phi-1) / 12}$ implies $A \leq x^{\phi}$. Since every $u$ in $S_{\phi}$, except at most 2 , is in $T(a, b)$ for some positive integers $a$ and $b$, we deduce that the number of elements of $S_{\phi}$ is

$$
\begin{aligned}
& \ll \sum_{a \leq A} \sum_{b \leq A} t(a, b)+x^{\phi} A^{-1} \\
& \ll y x^{(1-8 \phi) / 2} \sum_{a \leq A} \sum_{b \leq A}(a+b)+x^{(1-7 \phi) / 2} \sum_{a \leq A} \sum_{b \leq A} a b(a+b)+x^{\phi} A^{-1} \\
& \ll y x^{(1-8 \phi) / 2} A^{3}+x^{(1-7 \phi) / 2} A^{5}+x^{\phi} A^{-1} .
\end{aligned}
$$

Now,

$$
\begin{aligned}
A & =\min \left\{x^{(9 \phi-1) / 12}, y^{-1 / 4} x^{(10 \phi-1) / 8}\right\} \\
& = \begin{cases}x^{(9 \phi-1) / 12} & \text { for } \phi \geq(6 \gamma+1) / 12 \\
y^{-1 / 4} x^{(10 \phi-1) / 8} & \text { for } \phi<(6 \gamma+1) / 12 .\end{cases}
\end{aligned}
$$

Thus, for $\phi \geq(6 \gamma+1) / 12$, we obtain

$$
\left|S_{\phi}\right| \ll y x^{(1-7 \phi) / 4}+x^{(3 \phi+1) / 12} \ll x^{(3 \phi+1) / 12},
$$

and for $\phi<(6 \gamma+1) / 12$, we obtain

$$
\left|S_{\phi}\right| \ll y^{1 / 4} x^{(1-2 \phi) / 8}+y^{-5 / 4} x^{(22 \phi-1) / 8} \ll y^{1 / 4} x^{(1-2 \phi) / 8} .
$$

Hence, the result follows. 


\section{References}

[1] P. T. Bateman and E. Grosswald, On a theorem of Erdös and Szekeres, Illinois J. Math. 2 (1958), 88-98.

[2] M. Filaseta and O.Trifonov, On gaps between squarefree numbers, in: Analytic Number Theory, Proceedings of a Conference in Honor of Paul T. Bateman, Progr. Math. 85, B. Berndt et al. (eds.), Birkhäuser, Boston, 1990, 235-253.

[3] - - - On gaps between squarefree numbers II, J. London Math. Soc. (2) 45 (1992), $215-221$.

[4] D. R. Heath-Brown, Square-full numbers in short intervals, Math. Proc. Cambridge Philos. Soc. 110 (1991), 1-3.

[5] M. N. Huxley, The integer points close to a curve, Mathematika 36 (1989), 198215.

[6] C.-H. Jia, On squarefull integers in short intervals, Acta Math. Sinica 5 (1987), 614-621 (in Chinese).

[7] H. Liu, On square-full numbers in short intervals, ibid. 6 (1990), 148-164 (in Chinese).

[8] P. G. Schmidt, Zur Anzahl quadratvoller Zahlen in kurzen Intervallen, Acta Arith. 46 (1986), 159-164.

[9] -, Über die Anzahl quadratvoller Zahlen in kurzen Intervallen und ein verwandtes Gitterpunktproblem, ibid. 50 (1988), 195-201; Corrigendum, ibid. 54 (1990), 251254.

[10] P. Shiu, On squarefull integers in a short interval, Glasgow Math. J. 25 (1984), 127-134.

MATHEMATICS DEPARTMENT UNIVERSITY OF SOUTH CAROLINA COLUMBIA, SOUTH CAROLINA 29208 U.S.A.

E-mail: FILASETA@MATH.SCAROLINA.EDU
CENTER OF MATHEMATICS AND MECHANICS BULGARIAN ACADEMY OF SCIENCES P.O. BOX 373 1090 SOFIA, BULGARIA E-mail: DICE@BGEARN.BITNET

Received on 3.6.1993

and in revised form on 24.4 .1994 